\title{
Towards Open Collaborative Health Informatics - The Role of Free/Libre Open Source Principles
}

\section{Contribution of the IMIA Open Source Health Informatics Working Group}

\author{
T. Karopka' ${ }^{1}$ H. Schmuhl ${ }^{2}$, A. Marcelo ${ }^{3}$, J. Dal Molin 4 , G. Wright ${ }^{5}$ \\ ' IMIA Open Source Health Informatics Working Group Co-Chair \\ ${ }^{2}$ Center for Information Technology and Medical Engineering, University Hospital Heidelberg, Germany \\ ${ }^{3}$ University of the Philippines Manila, Philippines \\ ${ }^{4}$ e-cology corporation, Canada \\ ${ }^{5}$ IMIA Open Source Health Informatics Working Group Chair, Faculty of Health Sciences, Walter Sisulu \\ University, Mthatha, South Africa
}

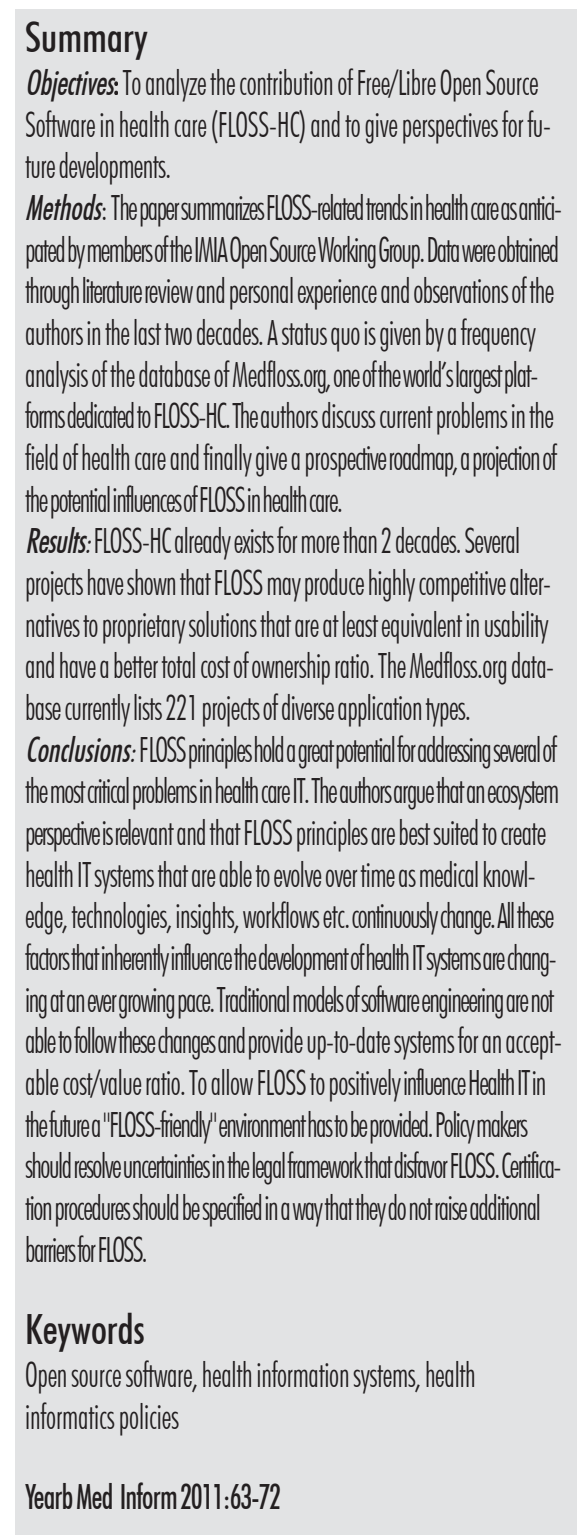

\section{Introduction}

The invention of the internet has introduced major changes in the way information and knowledge is distributed and processed which historically only may be compared with the invention of book-printing by Gutenberg. For the first time for mankind it has been possible to spread ideas and knowledge in a very effective way and to enable world-wide remote collaboration. With the internet not only has the method of distribution changed but also the way in which information is processed. This development has laid the foundation of the open source movement by providing unlimited communication and capabilities among newly formed worldwide, virtual communities. In the light of this development the authors of this paper will look at the role and the influence of FLOSS in health informatics in the past, present and future. After introducing the general concept of FLOSS the authors will give an overview of the development of FLOSS in health care by analyzing the data in the Medfloss.org database, one of the world's largest databases of FLOSS-HC. The activities of the IMIA OS WG will be presented as well as a strategy for the further development of IMIA OS WG. The paper will conclude with a discussion of how FLOSS may influence the future development of health informatics.

\section{Free/Libre Open Source Software (FLOSS)}

As the terms "Free Software" or "Open Source" are sometimes misleading, for the purposes of this article the term "Free/Libre Open Source Software" or in short "FLOSS" will be used. A brief explanation follows.

Share- or Freeware - software that is available for free, but often consists of proprietary/closed source code - is also often called "Free Software". In this context the word "free" simply means there is no fee and does not refer to freedoms that the user benefits from that are inherent to FLOSS. That is why the French word "libre", in reference to liberty or freedom, is included in the term FLOSS.

Furthermore the words "Open Source" can be misleading as it may only indicate that the source code is available and accessible whilst the free (re-)use of it may be limited or even forbidden by the license under which it is released.

A detailed and annotated definition of FLOSS can be found in "The Free Software Definition" [1] by the Free Software Foundation (FSF) and in "The Open Source Definition" [2] by the Open Source Initiative (OSI). Although they are not identical, as the definition of the FSF is a bit more strict whilst the OSI definition allows more licenses 
to fit into the "Open Source" paradigm. Both have their foundation in four essential user freedoms [1] formulated by Richard Stallman, free software activist and the founder of the FSF and the GNU project:

- "The freedom to run the program, for any purpose."

- "The freedom to study how the program works, and change it to make it do what you wish. (Access to the source code is a precondition for this.)"

- "The freedom to redistribute copies so you can help your neighbor."

- "The freedom to distribute copies of your modified versions to others. (By doing this you can give the whole community a chance to benefit from your changes. Access to the source code is a precondition for this.)"

It should be noted that "free" does not refer to the price, it does not mean that FLOSS is and can only be free of cost. The freedoms also assure that the software is available for commercial use, commercial development, and commercial distribution [1]. Diverse business models have been established around the FLOSS model [3]. But this is beyond the scope of this article, and will not be examined any further. Nevertheless, the availability of commercial FLOSS service and software providers within the domain of medicine and health care will be highlighted later on.

In this article the terms "Free Software", "Open Source" and "FLOSS" will be used synonymously and according to the definition of the FSF [1] and OSI [2]. The different types of Open Source software licenses will not be discussed. Both organizations provide a comprehensive and annotated overview of compatible and accepted licenses at their Web sites.

Today FLOSS has evolved from a "movement" to promote freedom in the software lifecycle to encompass a more diversified field of activities and is even seen by some people as the driving force in the software industry. A
European Study from Forrester Consulting commissioned by Bull states that "Open Source Becomes The Hidden Backbone Of The Software Industry, And Leads To A Paradigm Change In Enterprise IT" [4] and a report from Gartner states: "By 2012, 80 per cent of all commercial software will include elements of open-source technology. Many open-source projects are mature, stable and well supported. They provide significant opportunities for vendors and users to lower their total-cost-of-ownership and increase returns on investment. Ignoring this will put companies at a serious competitive disadvantage." [5].

\section{FLOSS in Health Care (FLOSS-HC)}

In spite of the recent success of FLOSS across industries this trend has yet to be seen in health IT. The reasons for this are many. In addition there is a general lack of research on this topic. A useful overview of the topic may be found in a white paper published by the AMIA OS WG [6]. The paper gives a good introductory overview of FLOSS and the specifics for FLOSS in health care with focus on the US and covers issues like licensing as well as benefits and challenges for FLOSSHC. The authors claim that an estimated number of $28,401,065$ patients have been entered into FLOSS systems throughout the US, whereof around 24 Million are entered into Federal Government sites.

In the following discussion we will analyze the data contained in the Web portal Medfloss.org [7], a database initiated originally by Schmuhl $[8,9]$ and motivated by Demski et al. [10] to provide a comprehensive and structured overview of FLOSS projects for the domains of medical informatics and health care. The platform is operated in cooperation with the Center for Information Technology and Medical Engineering of the University Hospi- tal Heidelberg, the Laboratory for Medical Information Systems (Medis) of the Helmholtz Zentrum München, the EFMI LIFOSS WG and the IMIA OS WG. Moreover it should offer a platform to foster the exchange of ideas, knowledge and experiences about these projects.

Information about relevant projects are stored and presented in a structured way. Besides a general description of the project, links are provided to resources such as the project homepage, downloads, support forum, service providers, publications, etc. the projects can be classified (tagged) in categories of application type, license, language, platform, client type, binary package, enterprise function, standard, programming language/toolkit and used database. The set of predefined terms in the category application type are based on Haux et al. [11] in section "3.5 Information Processing Tools in Hospitals". Selection of multiple terms is allowed as projects may belong to multiple application component types or cannot be clearly assigned to a single one. To take these aspects into account and enable non-IT-professional users to easily and quickly find projects of interest, a separate category "enterprise function" was introduced. The notion of enterprise function is as defined by Haux et al.[11].

As the number of projects and the amount of data is steadily growing and the information itself is rapidly changing it was decided to run Medfloss.org as a moderated open content platform. Any visitor can add or update data and through this form of participation it is expected that the best possible accuracy will be achieved in terms of quality and timeliness of information. To safeguard against Spam and off-topic content like Free-/Shareware with symptomatically closed sources, moderators review anonymously made changes and additions.

As of January 2011 the Medfloss.org database held 221 projects, 74 professional service providers and 97 references to scientific publications about 
listed projects [12]. Medfloss.org has been online since February 2010 and within the last 6 months experienced approximately 24,000 unique visitors and 225,000 page views according to its server logs.

In a recent study Zungi et al. [13] analyzed the functionalities of free and open electronic health record systems. All 12 examined software projects were already registered at Medfloss.org. This is seen as affirmation that an analysis of the data queried from the structured, relational database of Medfloss.org could provide a comprehensive if not representative overview of Open Source software within the domains of medical informatics and health care. Still it should be kept in mind that in the fast and ever changing environment of Open Source and health IT, it will never be possible to have a complete and up-todate listing of all existing projects.

The following paragraphs provide an overview of the characteristics of the registered projects by evaluating primarily the frequency distribution of tags (descriptive terms) within selected categories.

\section{Application Type}

In the category application type the most often used term is "Clinical EMR". EMR systems are classified at Medfloss.org in three different EMR sub-types: 56 (25\%) projects have been tagged as "Clinical EMR", 6 (3\%) as "Personal EMR" and 3 (1\%) as "Veterinary EMR". The second most frequently used term is "Framework \& Toolkit" which is divided into four sub-categories that reflect its main purpose: 46 (21 $\%)$ projects have been tagged as being a framework/toolkit for "Processing", 30 (14\%) for "Visualization", 24 (11\%) for "Analysis" and 8 (4\%) for "Simulation". Further popular terms are "Miscellaneous" with $36(16 \%)$ associated projects, "Hospital Management System" with $29(13 \%)$ and "Middleware" with $25(11 \%)$. Other typical application component types of hospital infor- mation systems can also be found: $16 /$ $8(7 / 4 \%)$ projects are tagged as "PACS Server (Picture Archiving and Communication System) Workstation/", 7 (3\%) as "CDMS (Clinical Data Management System)", 5 (2\%) as "LIS (Laboratory Information System)" and $3(1 \%)$ as "RIS (Radiology Information System)".

\section{Enterprise Function}

Looking at the frequency distribution of used terms within the category enterprise function, the three most often referenced terms are those describing the core functionality of EMR systems:

"Patient Management" is assigned to 80
(36\%) projects, "Record Management" to $75(34 \%)$ and "Clinical Documentation" to $74(33 \%)$. In the ranking these terms are followed by the tags "Data Processing" being associated with $44(20 \%)$ projects and "Image Processing" with $40(18 \%)$ that quite generally refer to essential functions provided by IT systems. When browsing through all 82 listed terms many important administrative and clinical enterprise functions are named, these are: 39 (18\%) tagged with "Data Visualization", 30 (14\%) with "Scheduling", $30(14 \%)$ with "Electronic Data Interchange", 27 (12\%) with "Medication Management" and "Billing" and 27 (12 $\%$ with "Statistical Analysis".

Table 1 Number of projects by application type (source: Medfloss.org)

\begin{tabular}{|c|c|c|c|}
\hline Rank & Application type & Count & in $\%$ \\
\hline 1 & EMR - Clinical EMR & 56 & 25 \\
\hline 2 & Framework \& Toolkit - Processing & 46 & 21 \\
\hline 3 & Miscellaneous & 36 & 16 \\
\hline 4 & Framework \& Toolkit- Visualization & 30 & 14 \\
\hline 5 & Hospital Management System & 29 & 13 \\
\hline 6 & Middleware & 25 & 11 \\
\hline 7 & Framework \& Toolkit - Analysis & 24 & 11 \\
\hline 8 & Software Library & 23 & 10 \\
\hline 9 & Pratice Management System & 20 & 9 \\
\hline 10 & PACS - PACS Workstation & 16 & 7 \\
\hline 11 & Disaster/Disease Management & 11 & 5 \\
\hline 12 & Framework \& Toolkit - Simulation & 8 & 4 \\
\hline 13 & PACS - PACS Server & 8 & 4 \\
\hline 14 & CDMS & 7 & 3 \\
\hline 15 & EMR - Personal EMR & 6 & 3 \\
\hline 16 & Public Health Management System & 5 & 2 \\
\hline 17 & US & 5 & 2 \\
\hline 18 & Telemedicine System & 5 & 2 \\
\hline 19 & RIS & 3 & 1 \\
\hline 20 & EMR - Veterinary EMR & 3 & 1 \\
\hline 21 & Web site & 2 & 1 \\
\hline 22 & Framework \& Toolkit & 2 & 1 \\
\hline 23 & Ontology & 1 & 0 \\
\hline 24 & DBMS & 1 & 0 \\
\hline
\end{tabular}


Table 2 Number of projects by enterprise function (abridged, source: Medfloss.org)

\begin{tabular}{|c|c|c|c|}
\hline Rank & Enterprise function & Count & in $\%$ \\
\hline 1 & PatientManagement & 80 & 36 \\
\hline 2 & Record Management & 75 & 34 \\
\hline 3 & Clinical Documentation & 74 & 33 \\
\hline 4 & Data Processing & 44 & 20 \\
\hline 5 & Image Processing & 40 & 18 \\
\hline 6 & Data Visualization & 39 & 18 \\
\hline 7 & Scheduling & 30 & 14 \\
\hline 8 & Electronic Data Interchange & 30 & 14 \\
\hline 9 & Medication Management & 27 & 12 \\
\hline 10 & Billing & 27 & 12 \\
\hline 11 & Application Integration & 26 & 12 \\
\hline 12 & Software Development & 25 & 11 \\
\hline 13 & Image Viewing & 24 & 11 \\
\hline 14 & Statistical Analysis & 24 & 11 \\
\hline 15 & Data Conversion & 21 & 10 \\
\hline 16 & Data Analysis & 19 & 9 \\
\hline 17 & Report Generation & 18 & 8 \\
\hline 18 & Image Communication & 18 & 8 \\
\hline 19 & Order Entry & 15 & 7 \\
\hline 20 & Data Management & 13 & 6 \\
\hline 21 & Prescribing & 13 & 6 \\
\hline 22 & Epidemiology & 13 & 6 \\
\hline 23 & Disease Management & 13 & 6 \\
\hline 24 & Image Management & 12 & 5 \\
\hline 25 & Data Validation & 11 & 5 \\
\hline$[\ldots]$ & {$[\ldots]$} & {$[\ldots]$} & {$[\ldots]$} \\
\hline
\end{tabular}

\section{License}

The category license, presented in Figure 1, draws a clear picture of which license is popular in the field of medical FLOSS. The majority of all projects that are in numbers 134 $(61 \%)$ are tagged to be licensed under a GNU General Public License (GPL). Only 33 (15\%) projects are licensed under the second most popular license, the GNU Lesser General Public License (LGPL), 24 (11\%)

\section{Standard}

The support of common standards is an essential requirement to deploy FLOSS project in heterogeneous IT environments as is typically found in health care institutions. In the category standard the most advanced and comprehensive interoperability standardization approach is IHE. The tag "IHE" is associated with at least $12(5 \%)$ projects at Medfloss.org.

The most popular standard is "HL7" which is supported by 40 (18\%) projects whereas the also text-based but universal XML data interchange format "XML" is referenced by at least $28(13 \%)$ projects. Standards in the area of medical imaging are also featured at Medfloss.org. 47 (21 \%) projects are tagged to support DICOM and $5(2 \%)$ the "NIfTI" data format.

Support of country specific standards or legislative regulations can also be found among the registered projects. For example $16(7 \%)$ projects are compliant to the requirements as defined by the American Health Insurance Portability and Accountability Action (HIPAA), 3 (1\%) implement the Swiss "TARMED" billing system and $2(1 \%)$ support the French "HPRIM" standard.

In addition to being able to tag projects within different categories, it is also possible to incorporate each one with other content types like references to scientific articles, professional service providers and more.

\section{Publications}

At the moment 97 references to scientific publications are registered at Medfloss.org, most of them are also indexed at Medline. These publications provide additional comprehensive insight as to the purposes, requirements, architectures, functionalities and experiences of Open Source software projects in health care. Furthermore it shows that FLOSS is not just about programming it is also of academic interest. Projects with most references in 


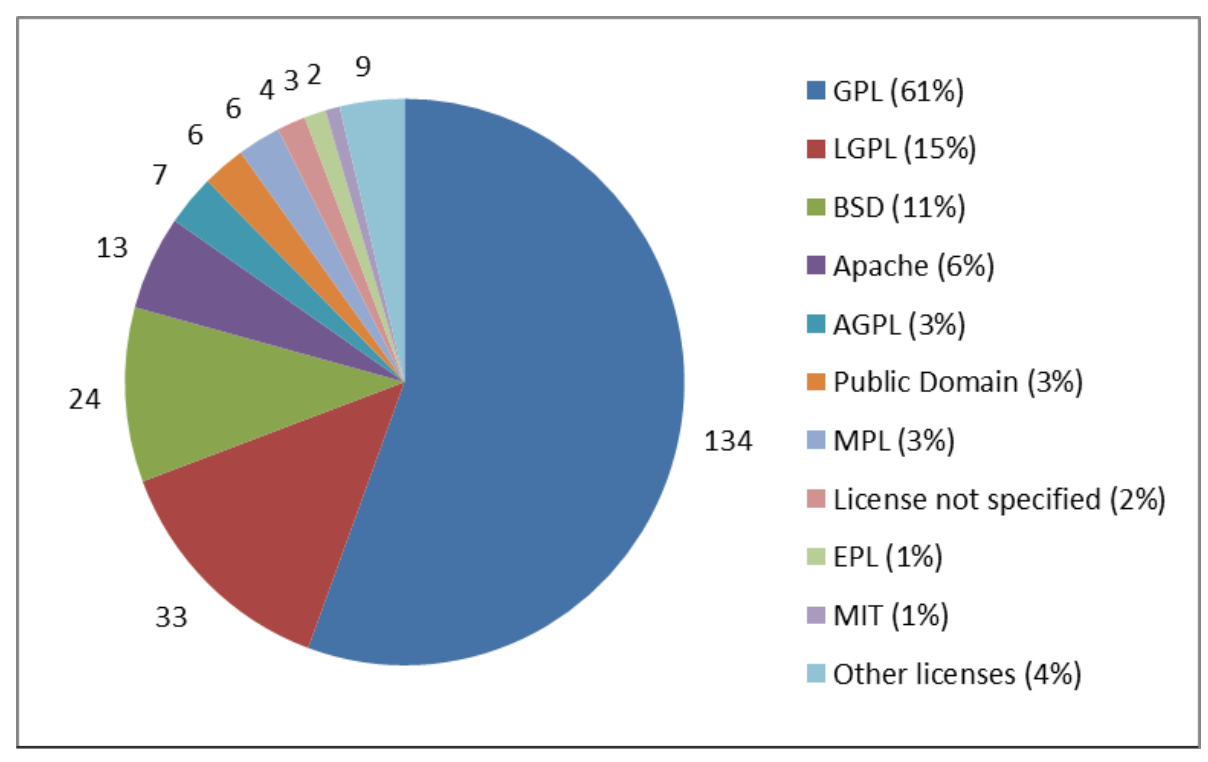

Fig. 1 Number of projects by license (source: Medfloss.org)

the Medfloss.org database are "OpenMRS" [14] and "Indivo" [15] of which everyone has 6 related publications registered closely followed by "OsiriX" [16] with 5. In total 63 projects are referred to by scientific articles.

\section{Professional Service Provider}

One of the key concerns when deploying FLOSS in productive environments is the availability of professional support. Although there exists for nearly every Open Source project a broad range of community - powered support through project specific mailing lists, discussion forums and wiki - like documentation platforms, this may not be sufficient to resolve all issues adequately and in a timely manner. To address this shortcoming a considerable quantity of companies offering professional services have been established within the area of medical FLOSS. For example for the project "OSCAR" [17] there are 8 service providers known, followed by the projects "OsiriX" [16] with 7 and "Mirth" [18] with 5 . In total there are 74 service providers registered at Medfloss.org that offer their services for at least 53 projects. The presence of a company does not only guarantee support, it is also indicative of the maturity level of the project itself as companies are able to build a revenue generating businesses upon it.

\section{Towards Health Informatics 3.0 - A prospective View on FLOSS in Health Care}

There is no doubt that there is a global change in the mindset of knowledge gatekeepers and the expection of open access to knowledge which has become part of the internet revolution. The way in which FLOSS will contribute to or influence the field of medical informatics depends on a couple of driving forces namely development of new technologies (e.g. mobile technologies), legal frameworks, certification procedures, funding policies and financial constrains to name just a few. The future challenges for health informatics also depend on the local context. While most of the industrial- ized countries have to cope with rising costs in their health care system, shortage of human resources, an aging population with multi-morbidity and chronic disease partly lifestyle related, most of the developing countries are fighting against HIV/Aids, malaria and tuberculosis. The lack of skilled human resources is much greater in the developing world and there are additional challenges like poverty, lack of infrastructure and access to energy [19].

In [20] Haux suggests that in the near future

(a) Health has to be considered more and more as an integral and continuous part of life

(b) Medical informatics is addressing both, health professionals and individuals/consumers

(c) The individual is being at the center of research

(d) Research, education and practice may shift more and more from local to global activities

With these hypotheses in mind we will look at challenges and prospective developments in FLOSS-HC from different perspectives and contexts.

\section{FLOSS in Low Resource Settings}

In low resource settings the FLOSS methodology is gaining attraction due to the lower Total Cost of Ownership (TCO). NGOs are starting to look at FLOSS as a means to reuse successful implementations while keeping the ability to adapt to local requirements. In this context FLOSS is also used in the sense of an "ethical" correct or good way of supporting developing nations. Most of the stakeholders share the values of the social movement that originated in the free software movement. The Open Medical Record System (OpenMRS) [14] is one of the success stories where the FLOSS methodology combined with funding and community building has led to a sustainable and growing community of developers and users [21]. 
In developing countries, these challenges are compounded by the scarcity of financial resources for the infrastructural components of health information systems. Having no access to hardware and the information systems, capacities for learning health informatics are also limited. If technology is introduced, poor organizational and expertise capacity prevents the technology from prospering. Recent developments in the application of mobile phones as computing devices in healthcare may allow developing countries to overcome some of these infrastructure barriers. mHealth is seen as one of the most promising technologies for developing countries [22].

\section{Support of Open Standards}

It is often not sufficient to have an open standard. Today's standards in health care IT are extremely complex. Two different implementations of an open standard are often not 100\% compatible. Additionally it is very costly to implement complex standards. Reference implementations, component building blocks and common modular infrastructure software could be a valuable contribution towards interoperable systems in healthcare IT. This would also lower overall costs. All stakeholders would benefit from such an initiative. Examples of already well-established, FLOSS based interoperability frameworks are the Open Health Tools [23], Mirth [18], Open eHealth Integration Platform [24] and ConnectOpenSource [25].

\section{Political Support and the Need for Open Source Governance}

Recently several governments have worked out policies to foster FLOSS in the public sector. South Africa and Australia are amongst those who now demand that Government funded systems are Open Source, whilst the UK has asked that it be considered in pro- curement processes. Also local governments and regions are supporting FLOSS initiatives. For example the Paris Region supports the Open World Forum [26]. The Brazilian government has set up the Brazilian Public Software Portal [27] dedicated to fostering the use of FLOSS. The European Commission has set up the Open Source Observatory and Repository for European public administrations (OSOR) [28] as a platform for exchanging information, experiences and FLOSS-based code for use in public administrations. For a recent review on the status of open source software in the public sector please see [29]. A similar engagement in the field of health care could greatly enhance the positive impact of FLOSS in health care. Non-governmental organizations (NGOs) play a vital role in the FLOSS-HC ecosystem. NGOs and foundations are more and more looking at FLOSS to achieve a better "health for money" ratio in their funding strategy. IDRC Canada and Rockefeller Foundation have supported OpenMRS [14]. RapidSMS [30], UNICEF's open source framework for mobile data collection, was designed to be customized for the challenges of governments, multilateral, international- and non-government organizations, and development practitioners: working effectively in spite of geographical remoteness of constituents, limited infrastructure (roads, electricity), and slow data collection. MEDICAL [31], a universal hospital information system specifically designed for low resource settings initiated by GNU Solidario [32] is now supported by the United Nations University. Despite the very good results from these projects there is still too little cooperation and coordination among FLOSS health projects. The positive impact could be significantly much bigger if different open source projects would work together towards a modular, component based open source platform for health care. This requires open source governance. IMIA
OS WG is working towards this goal in bringing together key stakeholders in FLOSS-HC.

\section{Economic Evidence}

It is generally accepted that the cost of implementing a system is similar regardless of whether it is a proprietary or FLOSS solution. So is cost saving through FLOSS a myth? There is no doubt that when commercial companies who provide proprietary code go out of business the subsequent cost of not being able to develop or maintain the software may be beyond affordable by those who have purchased the system. In some other areas are true cost savings. For example the cost to a large organization when using Open Source operating systems and associated software could drastically reduce yearly cost, due to the high number of workstation and related license costs. A university for example could save millions of dollars and in the developing world this would significantly reduce operating costs.

From an economic point of view it simply makes sense to collaboratively develop software health care systems particularly as these are directly or indirectly paid for by the tax payer in most countries. FLOSS provides a mechanism to jointly develop a common framework and then concentrate on innovation. An impressive example is the VistA system [33] from the U.S. Department of Veterans Affairs, a system which has been developed in a collaborative way for over twenty years and whose code base is available under different open source licenses. A recent review in Health Affairs states that: "The potential value of the VA's health IT investments is estimated at $\$ 3.09$ billion in cumulative benefits net of investment costs." [34].

Many companies have not realized that FLOSS lowers barriers to entry and enables Small and Medium companies (SMEs) to actively participate in the market. SMEs usually do not have the 
ability to set up a project on their own that requires several person years of work. On the other hand FLOSS solutions are much more flexible and could do support, configuration and adaptation of software. The prevailing business model for proprietary software and its continuing trend to address global markets is to sell software as it is without doing adaptations for local requirements, this leads to very inflexible systems and the users often get stuck into this innovation trap. IT companies can change their business model and instead of selling licenses they should provide service, support, customization and consulting services. The collaborative development approach saves time and money and gives room for concentrating on innovation and not spending time for developing commodities. For an economic motivation of open source see e.g. the work of Perens [35] or Riehle [36, 37].

\section{Personal Health Systems - Smart Homes and Ambient Assisted Living}

One of the main challenges for the industrialized nations is demographic trends. The increase in life expectancy and the simultaneous growth in the number of age related diseases call for a close collaboration among all stakeholders involved in the care process for the elderly. To cope with this situation a new field of research has been created which is called Ambient Assisted Living (AAL). The term Ambient Assisted Living was created in Germany and subsequently used for the Joint Program for Ambient Assisted Living at the EU level. The main goal of AAL is to assist elderly people by providing solutions that enable them to live independently as long as possible in their own homes. Research in this area overlaps with other research areas/communities, e.g. telecommunication, sensor technologies, home automation to name a few from the technical side. A challenge is to bring these different fields together and provide an infrastructure to assemble devices and services to complex systems. Several EU-funded projects have developed frameworks for AAL: Persona [38], OASIS [39], MPOWER [40], MonAMI [41], SOPRANO [42], VAALID [43]. Most of these projects release their code or parts of it under an open source license. To reuse as much as possible of the output of these projects the UniversAAL project [44] has been created with the main objective to make it technically feasible and economically viable to conceive, design and deploy innovative new AAL services. The UniversAAL project draws from the contributions of the earlier projects and aims to provide a universal platform which is available under an open source license. To provide a meeting point able to catalyze interest and efforts around AAL, the universAAL project created the non-profit AAL Open Association (AALOA) [45].

\section{Health Informatics Infrastructure Based on FLOSS}

Aside from AAL the trend towards the establishment of regional, multi-institutional, patient-centered, electronic health record (PEHR) as proposed by Heinze et al. [46] also reflects Haux's hypotheses [20] and is another example in which the overall goal can only be reached by the utilization of FLOSS. No matter to which extent and in which structure a PEHR is established, the critical mission is always to integrate medical data that is coming from multiple heterogeneous sources. Each connected health care institution has its own information systems that are quite different from one another and may not or only to a certain extent support standardized interfaces. To address this natural but serious short coming, middleware is utilized to enable proper interoperability between the institution-specific information systems. Beside the actual transformation of data into a common format, also additional central components are needed to fulfill the overall requirements of a PEHR system such as data privacy and security, availability of registries and repositories to link and/ or store the payload and administrative data of the actual PEHR system to name just a few. Although the connected EMR and PHR systems may vary a lot depending on the specific scenario and its stakeholders, the overall requirements still remain always the same. As proposed by Sfakianakis et al. [47] to equally enable all system vendors and health care institutions within a regional network to connect their systems to the PEHR, the only way is to provide the middleware and its core components under a FLOSS license. Only in this way can access be guaranteed to interface definitions, and how the central software components work, and is it possible to modify or extend functionality without the encumbrance of proprietary licenses. Furthermore, the resulting FLOSSbased infrastructure can be re-used without any license cost in other regional health care networks, so that they do not have to start from scratch and might contribute additional, valuable features to existing components. As for now many of the PEHR projects are initiated and funded by public authorities it is just natural that the resulting software is released as FLOSS. So also other projects and initiatives can profit from the outcome and tax payers do not have to pay for the same thing again and again. A good example for an open source infrastructure is the U.S.-based ConnectOpenSource project [25] that aims to provide middleware to connect health information exchanges in the National Health Information Network (NHIN).

\section{Research and Education: FLOSS in Undergraduate Medical and Nursing Education}

Health informatics has started to become integrated in medical and nurs- 
ing curricula of many countries. But the challenge of providing meaningful laboratory exercises to the students have been challenging for several reasons. One example is that informatics laboratories often require the purchase of expensive equipment, and software licenses which can reach prohibitive levels beyond the budget of small schools who in turn often pass this cost to the students. In the Philippines, FLOSS is being used as the software of choice for teaching concepts in health informatics. Students are able to understand the principles of health information management through actual use of FLOSS electronic medical records (OpenMRS [14]), course management systems (Moodle [48]), and telemedicine platforms (iPath [49]). At that level they are able to learn electronic medical records and the ancillary non-technical aspects such as ethical management of information privacy, and security. This prepares the doctors and nurses of the future to understand the benefits and risks of EMRs without the environmental pressures of litigation or malpractice. It also introduces the concepts at a much earlier stage of their career and prepares them for the actual use of EMRs in their eventual practice. FLOSS makes it possible for underbudgeted teaching institutions to upgrade their content without incurring substantial costs.

\section{The Role of the IMIA Open Source Working Group}

The IMIA Open Source Working Group was formed in 2002 with the aim to provide a forum for discussion and for a collaborative, non-judgmental work environment to explore, and where appropriate promote and facilitate, the application of FLOSS solutions within health, healthcare and health informatics.

IMIA OS WG seeks to bring together experts and interested individuals from a wide range of health profes- sions and with a range of interests in the potential application of FLOSS solutions within their domains of expertise and to explore the implications of the FLOSS approaches for all aspects of IMIA's areas of interest.

FLOSS principles are well suited to making knowledge freely available and accessible and can be considered as a role model for "Open Access" thus supporting the evolution of medical informatics. Currently the knowledge about FLOSS as a methodology as well as the medical knowledge that is concealed in diverse FLOSS-HC applications and projects is dispersed over several projects, researchers, applications, communities etc. This knowledge has to be integrated and made available through one focal access point where all stakeholders may enter the virtual space of FLOSS-HC. The Medfloss.org website is intended to evolve towards this goal.

IMIA OS WG is committed to disseminate knowledge about FLOSS-HC in the health care community through organization of workshops and conferences and through participation of WG members in conferences related to health care with the mission to spread knowledge about the prospects of FLOSS-HC [50], [51], [52], [53], [54], [55], [56]. The FLOSS WG, being made up of thought leaders and influential educators and researchers, can promote the benefits of FLOSS to their network of stakeholders.

We foresee that health informatics 3.0 will be increasingly influenced by the initiative of individuals and small groups. In the past only big companies, organizations or governments could have a real impact on the development of a technology or a standard. Social networking allows motivated people to connect and coordinate their actions. A very impressive example is the emergence of humanitarian FLOSS, where people use ICT in crisis management (e.g. Crisis Commons [57]) as well as the mobile community (e.g. MobileActive.org [58], where people meet that propose mobile technology for social change).
Social networking has emerged only recently and is an extremely powerful tool to empower individuals and small, often dispersed groups and to allow them to coordinate their actions. Virtual collaboration and communitybased work is natural and the driving force of FLOSS projects since its early beginning. By the evolvement of social networks new helpful means are available to further foster this process, beside the long-serving capabilities of mailing-lists, forums and IRC-channels, just to name a few. In healthcare this has manifested itself in collaborative patient portals where information on how to manage health issues is freely exchanged.

\section{Conclusions}

It is perceived that FLOSS has the potential to deliver better value for money, higher quality software, more secure, stable, reliable and flexible network solutions. As stated earlier this has been publically acknowledged by many governments as part of their IT policy. Many Health services IT systems are based on the principles of a service-oriented architecture which allows disparate clinical information systems to be seamlessly integrated over a network according to a set of interfaces and protocols. Cloud systems and thin clients are also reliant on open source software and open standards. Is the continued growth of these techniques and innovations possible without the participation of FLOSS communities? It is hard to imagine the establishment and exponential growth of the WWW without the omnipresence of FLOSS technologies and ideologies that formed the base of this development.

The analysis of the Medfloss.org database shows that the availability and exploitation of specialized Open Source software in the domains of medical informatics and health care is not in its infancy. It has been actively growing already for a long time, is well 
established, and the available projects offer broad functionality for variety of areas of application. With the Medfloss.org platform at hand it is possible to present existing projects in a structured way and to simplify the search for suitable projects.

As Medfloss.org is not the only listing of medical FLOSS on the Internet, the interested reader should also take a look at the following sources [59], [60], [61]. A special focus should also be put at the Debian Med project [62] that is packaging medical FLOSS for the Debian and other Debian-based Linux distribution like Ubuntu. Besides providing the community with the very valuable capability to perform one-click installations of many projects, they also maintain a comprehensive list of medical FLOSS at [63]. Meanwhile also the OpenSUSE and the Fedora communities have started similar initiatives to pack projects for their distributions [64], [65].

In advanced economies the lower TCO is not the key argument for looking at FLOSS methodologies. The strongest arguments supporting FLOSS are the flexibility, the reuse of complex systems and the ability to use a common framework while investing in features for differentiation and user driven, rapid innovation. In this context Open Source is more and more used as a business model. The main barriers for adoption of FLOSS applications are not technical but are often in the realm of legal requirements e.g. certification, lack of professional support services and policies that disfavor the use of open source. In a recent study Paré et. al. found that "key factors for not adopting an open source solution were closely linked to the orientations of ministry level policy makers and a seeming lack of information on the part of operational level IT managers concerning commercially oriented open source providers" [66].

A "healthy" and sustainable FLOSS$\mathrm{HC}$ ecosystem should consist of the following principal constituents: FLOSS projects/products, community (developers, users), support companies, FLOSS experts and advocates, FLOSS supporters among health IT decision and policy makers. Given these ingredients FLOSS-HC should flourish and if applied in a proper way will benefit all stakeholders in the ecosystem.

\section{References}

1. The Free Software Definition. [cited 2011/01/29]; Available from: http://www.gnu.org/philosophy/ free-sw.html.

2. The Open Source Definition (Annotated), Version 1.9. [cited 2011/01/29]; Available from: http:// www.opensource.org/osd.html.

3. Riehle D. The Commercial Open Source Business Model. In: Nelson ML, Shaw MJ, Strader TJ, editors. Value Creation in EBusiness Management: Springer Berlin Heidelberg; 2009. p. 18-30.

4. Commissioned Study Shows Open Source Paves The Way For The Next Generation Of Enterprise IT. [cited 2011/01/29]; Available from: http:// www.wcm.bull.com/internet/pr/ rend.jsp?DocId=412289\&lang=en.

5. Gartner Highlights Key Predictions for IT Organisations and Users in 2008 and Beyond. [cited 2011/01/29]; Available from: http:// www.gartner.com/it/page.jsp?id=593207.

6. Valdes I. Free and Open Source Software in Healthcare 1.0. [cited 2011/01/29]; Available from: https://www.amia.org/files/Final-OSWG\%20White\%20Paper_11_19_08.pdf.

7. Medfloss.org - Medical Free/Libre and Open Source Software. [2011/01/29]; Available from: http://www.medfloss.org/.

8. Schmuhl H. Medfloss.org - A comprehensive platform for medical free/libre and open source software. 2010 [cited 2011/01/29]; Available from: http://www.apfelkraut.org/download/ Medfloss_Med-e-Tel_2010_H_Schmuhl.pdf.

9. Schmuhl $\mathrm{H}$. What Else Is Going On? - Building a Project Database. 2008 [cited 2011/01/29]; Available from: http://www.apfelkraut.org/ download/med-e-tel_oss_db_h_schmuhl.pdf.

10. Demski H, Hildebrand C, Jossif A, Engelbrecht $\mathrm{R}$, editors. Information on Open Source Solutions in Healthcare - the OSSHealth Repository. MedE-Tel; 2010; Luxembourg.

11. Haux R. Strategic information management in hospitals : an introduction to hospital information systems. New York: Springer; 2003.

12. Statistics about medfloss.org. [cited 2011/01/ 29]; Available from: http://www.medfloss.org/ node/474.

13. Flores Zuniga AE, Win KT, Susilo W. Functionalities of free and open electronic health record systems. Int J Technol Assess Health Care. 2010 Oct;26(4):382-9.

14. OpenMRS - Open source health IT for the planet. [cited 2011/01/29]; Available from: http:// openmrs.org/.
15. The Indivo Personally Controlled Health Record. [cited 2011/01/29]; Available from: http:// indivohealth.org/.

16. OsiriX Imaging Software. [cited 2011/01/29]; Available from: http://www.osirix-viewer.com/.

17. OSCAR Canada Users Society. [cited 2011/01/ 29]; Available from: http://www.oscarcanada.org/.

18. Mirth Connect - Mirth Corporation. [cited 2011/ 01/29]; Available from: http://www. mirthcorp. $\mathrm{com} /$ community/mirth-connect.

19. Mars M. Health capacity development through telemedicine in Africa. Yearb Med Inform 2010:87-93.

20. Haux R. Medical informatics: past, present, future. Int J Med Inform 2010 September;79(9):599-610

21. Seebregts CJ, Mamlin BW, Biondich PG, Fraser HSF, Wolfe BA, Jazayeri D, et al. Human Factors for Capacity Building. Lessons learned from the OpenMRS Implementers Network. Yearb Med Inform 2010:13-20.

22. Akter S, Ray P. mHealth - an Ultimate Platform to Serve the Unserved. Yearb Med Inform 2010:94100.

23. Open Health Tools. [cited 2011/01/29]; Available from: http://www.openhealthtools.org/.

24. Open eHealth Integration Platform. [cited 2011/ 01/29]; Available from: http://repo. openehealth.org/.

25. NHIN CONNECT Community Portal. [cited 2011/01/29]; Available from: http://www. connectopensource.org/.

26. Open World Forum 2010. [cited 2011/01/29]; Available from: http://www.openworldforum.org

27. Portal do Software Público Brasileiro. [cited 2011/01/29]; Available from: http://www. softwarepublico.gov.br/.

28. OSOR.eu - Open Source Observatory and Repository. [cited 2011/01/29]; Available from: http://www.osor.eu/.

29. Report on the International Status of Open Source Software 2010. [cited 2011/01/29]; Available from: http://observatorio.cenatic.es/index.php? option=com_content\&view=article\&id=666:reporton-the-international-status-of-open-sourcesoftware-2010\&catid $=13$ :empresas \&Itemid $=23$.

30. RapidSMS / SMS Application Framework. [cited 2011/01/29]; Available from: http://www. rapidsms.org/.

31. Medical - The Universal Hospital and Health Information System. [cited 2011/01/29]; Available from: http://medical.sourceforge.net/.

32. GNU Solidario : Making a difference with Free Software. [cited 2011/01/29]; Available from: http://www.gnusolidario.org/en/index.html.

33. VistA - Vistapedia. [cited 2011/01/29]; Available from: http://vistapedia.net/

34. Byrne CM, Mercincavage LM, Pan EC, Vincent AG, Johnston DS, Middleton B. The value from investments in health information technology at the U.S. Department of Veterans Affairs. Health Affairs (Project Hope). 2010 April;29(4):629-38.

35. Perens B. Bruce Perens - The Emerging Economic Paradigm of Open Source. [cited 2011/01/29]; Available from: http://perens.com/works/articles/ Economic.html.

36. Riehle D. The Economic Motivation of Open Source Software: Stakeholder Perspectives. Computer 2007 April;40:25-32. 
37. Riehle D. The Single-Vendor Commercial Open Source Business Model. Information Systems and e-Business Management: Springer Verlag; 2011.

38. PERSONA Project Portal. [cited 2011/01/29]; Available from: http://www.aal-persona.org/.

39. OASIS - Open architecture for Accessible Services Integration and Standardization. [cited 2011/01/ 29]; Available from: http://www.oasis-project.eu.

40. MPOWER. [cited 2011/01/29]; Available from: http://www.sintef.no/mpower.

41. MonAMI. [cited 2011/01/29]; Available from: http://www.monami.info/.

42. SOPRANO - Service-oriented Programmable Smart Environments for Older Europeans. [cited 2011/01/29]; Available from: http://www.sopranoip.org/.

43. Vaalid - Accessibility and Usability Validation Framework for AAL Interaction Design Process. [cited 2011/01/29]; Available from: http:// www.vaalid-project.org/.

44. UniversAAL. [cited 2011/01/29]; Available from: http://www.universaal.org/.

45. AAL Open Association - AALOA. Available from: http://www.aaloa.org/.

46. Heinze O, Bergh B. Establishing a Personal Electronic Health Record in the Rhein-Neckar Region. Slovenian Medical Informatics Association 2009; 14(1-2):3-9.

47. Sfakianakis S, Chronaki CE, Chiarugi F, Conforti F, Katehakis DG. Reflections on the role of open source in health information system interoperability. Yearb Med Inform 2007:50-60.

48. Moodle.org: open-source community-based tools for learning. [cited 2011/01/29]; Available from: http://moodle.org/.

49. iPath-Network. [cited 2011/01/29]; Available from: http://www.ipath-network.com/.
50. International Workshop on e-Health in Emerging Economies. [cited 2011/01/29]; Available from: http://www.iweee.org/.

51. The First International Workshop on Open Source in European Health Care: The Time is Ripe OSEHC 2009. [cited 2011/01/29]; Available from: http://www.biostec.org/Biostec2009/ OSEHC.htm.

52. 2nd International Workshop on Open Source in European Health Care: Crossing the Borders OSEHC 2010. [cited 2011/01/29]; Available from: http://www.biostec.org/Biostec2010/ OSEHC.htm.

53. EFMI STC 2008: Open Source and Healthcare in Europe - Time for Leading Edge Ideas?, September 2008, London, UK. [cited 2011/01/29]; Available from: http://www.differance-engine.net/ efmistc2008/index.php.

54. Special track on FLOSS-HC projects and initiatives at Med-e-Tel 2010. [cited 2011/01/29]; Available from: http://www.medetel.lu/index. php?rub=educational_program \&page= benefits_of_open_source_software_in_health_care.

55. Special track on FLOSS-HC projects and initiatives at Med-e-Tel 2011. [cited 2011/01/29]; Available from: http://www.medetel.lu/index. php?rub=educational_program\&page $=$ benefits_of_open_source_software_in health_care_2011.

56. Murray PJ, Wright G, Karopka T, Betts H, Orel A. Open source and healthcare in Europe - time to put leading edge ideas into practice. Stud Health Technol Inform 2009;150:963-7.

57. CrisisCommons. [cited 2011/01/29]; Available from: http://crisiscommons.org/.

58. MobileActive.org | A global network of people using mobile technology for social impact. [cited 2011/01/29]; Available from: http://www. mobileactive.org/.

59. List of open source healthcare software - Wikipedia, the free encyclopedia. [cited 2011/01/29]; Available from: http://en.wikipedia.org/wiki/ List_of_open_source_healthcare_software.

60. SourceForge.net: Software Search "Medical Science Apps." [cited 2011/01/29]; Available from: http://sourceforge.net/softwaremap/ ?\&fq[]=trove $\% 3 \mathrm{~A} 266$.

61. Janamanchi B, Katsamakas E, Raghupathi W, Gao W. The state and profile of open source software projects in health and medical informatics. Int J Med Inform 2009 Jul;78(7):457-72.

62. Debian Med. [cited 2011/01/29]; Available from: http://www.debian.org/devel/debian-med/.

63. Debian Med Task Page. [cited 2011/01/29]; Available from: http://debian-med.alioth. debian.org/tasks/.

64. Portal:Medical OpenSUSE. [cited 2011/01/29]; Available from: http://en.opensuse.org/ Portal:Medical.

65. SIGs/FedoraMedical - FedoraProject. [cited 2011/ 01/29]; Available from: http://fedoraproject.org/ wiki/SIGs/FedoraMedical.

66. Pare G, Wybo MD, Delannoy C. Barriers to open source software adoption in Quebec's health care organizations. J Med Syst 2009 Feb;33(1):1-7.

\section{Correspondence to:}

Thomas Karopka

Stralsunder Str. 18

18445 Prohn

Germany

E-mail: TKaropka@gmail.com 